\title{
LETTER OPEN \\ Regulation of SKP2 protein stability by heat shock protein 90 chaperone machinery
}

\author{
Signal Transduction and Targeted Therapy (2021)6:276
}

\section{Dear Editor,}

$\mathrm{S}$ phase kinase-associated protein 2 (SKP2), a substrate recognition component of the $\mathrm{SCF}^{\mathrm{SKP} 2}$ ubiquitin ligase complex, plays an oncogenic role in tumorigenesis by targeting a variety of tumor suppressors (e.g., p21, p27, and p130) for ubiquitination and subsequent degradation. ${ }^{1}$ As a well-characterized oncoprotein, the aberrant expression and dysregulation of SKP2 are frequently observed in different human cancers. Given its critical role in governing tumorigenesis and progression, SKP2 has emerged as a potential pharmacological target for anticancer therapy. ${ }^{1}$

Heat shock protein 90 (HSP90) is an evolutionarily conserved molecular chaperone that participates in stabilizing and activating $>200$ proteins termed HSP90 client proteins. ${ }^{2}$ In addition, HSP90 is highly expressed in various tumors compared with normal tissues to maintain the stabilization of numerous client oncoproteins. Therefore, several small-molecule inhibitors (e.g., 17-AAG and STA9090) that target HSP90 to inhibit its ATPase activity have been developed as potential anticancer drugs and undergo clinical trials in various human cancers. ${ }^{2}$ Given that SKP2 is sophisticatedly regulated in human tumors, whether it can be directly regulated by HSP90 remains poorly understood.

In order to examine whether SKP2 is regulated by HSP90 chaperone machinery, two HSP90 small-molecule inhibitors, 17AAG and STA-9090, were applied to treat several human cancer cell lines (A549, H1299, MDA-MB-231, and SW480). It was observed that treatment with 17-AAG or STA-9090 downregulated SKP2 protein expression, and subsequently led to the accumulation of p27, the most classical substrate of SKP2 (Fig. 1a, b). AKT, a known client protein for HSP90, was used as a positive control of HSP90 inhibition (Fig. 1a, b). As for the mechanism investigation, we found that treatment of 17-AAG or STA-9090 had little effect on SKP2 mRNA levels (Supplementary Fig. S1). To further ascertain how HSP90 inhibitors downregulate SKP2 expression, we examined the effect of 17-AAG on the stability of SKP2. To this end, we applied cycloheximide to block new protein synthesis and detected SKP2 degradation with or without 17-AAG treatment. As shown in Fig. 1C, 17-AAG treatment shortened the half-life of SKP2 (Fig. 1c). In order to verify the HSP90-SKP2-p27 axis, we further examined whether SKP2 rescue could abrogate 17-AAG/ STA-9090-induced upregulation of p27, and found that SKP2 overexpression partially rescued the p27 accumulation upon treatment of 17-AAG or STA-9090 (Supplementary Fig. S2). Collectively, these data demonstrate that HSP90 inhibitors promote SKP2 degradation and subsequent p27 accumulation.

We further determined whether HSP90 inhibitor-induced degradation of SKP2 was regulated through ubiquitin-proteasome pathway or lysosome degradation pathway. As the result shown, STA-9090-induced protein degradation of SKP2 was obviously blocked by MG132 (a classical proteasome inhibitor), but neither by CQ (chloroquine) nor bafilomycin A1 (both are classical lysosomal

\author{
; https://doi.org/10.1038/s41392-021-00624-1
}

inhibitors; Fig. 1d, e and Supplementary Fig. S3), indicating that STA-9090 treatment induces SKP2 degradation in a proteasomedependent manner. Moreover, we examined whether SKP2 underwent ubiquitination upon treatment with HSP9O inhibitors, and found that the levels of SKP2 poly-ubiquitination were significantly enhanced upon treatment with either 17-AAG or STA-9090 (Fig. If, $\mathrm{g}$ and Supplementary Fig. S4), suggesting that HSP90 inhibitors induce SKP2 ubiquitination and subsequently proteasomemediated degradation. Meanwhile, we tested whether HSP90 isoforms bind to SKP2. As shown in Fig. 1h, SKP2 interacted with both HSP90a and HSP90 $\beta$ under ectopic overexpression condition (Fig. 1h). Furthermore, in vitro purified GST-SKP2 effectively pulled down both His-HSP9Oa and His-HSP90ß (Fig. 1i), further indicating that HSP90 directly binds to SKP2 and regulates its stability.

The $\mathrm{C}$ terminus of $\mathrm{Hsc} 70$-interacting protein (CHIP, also known as STUB1) is one of the major E3 ubiquitin ligases involved in the HSP90 chaperone system. ${ }^{3}$ We thus tested whether CHIP serves as an E3 ligase mediating SKP2 degradation upon the treatment of HSP90 inhibitors, and found that CHIP knockdown did not rescue 17-AAG or STA-9090-induced SKP2 degradation (Fig. $1 \mathrm{j}$ and Supplementary Fig. S5a). Likewise, ectopic overexpression of CHIP had no effect on the SKP2 expression (Supplementary Fig. S5b). Thus, these results suggest that HSP90 inhibitor-induced SKP2 degradation is independent of CHIP. Besides CHIP, Cullin5 (CUL5) E3 ligase also mediates ubiquitin-dependent degradation of HSP90 client proteins. ${ }^{3}$ However, CUL5 knockdown also had no effect on HSP90 inhibitor-induced SKP2 degradation (Fig. 1k and Supplementary Fig. S5c). Furthermore, we examined whether other Cullin-Ring E3 ligases (CRLs) contribute to HSP90 inhibitorinduced SKP2 degradation. To this end, we treated cells with MLN4924, the specific NEDD8-activating enzyme inhibitor that inhibits Cullin neddylation and induces CRLs inactivation, and found that MLN4924 treatment did not compromise STA-9090induced SKP2 degradation (Supplementary Fig. S5d).

Since $\mathrm{CDH} 1$ is a known E3 ligase to mediate SKP2 destruction, ${ }^{4}$ thus we tested whether $\mathrm{CDH} 1$ is responsible for HSP90 inhibitorinduced SKP2 degradation. It was observed that knockdown of $\mathrm{CDH} 1$ induced SKP2 accumulation (Supplementary Fig. S6, lane 1 vs lane 2), but only modestly compromised HSP90 inhibitor-induced SKP2 degradation (Supplementary Fig. S6), indicating that HSP90 inhibitor-induced SKP2 degradation was regulated by an unknown E3 ligase other than $\mathrm{CDH} 1$. In order to identify the putative E3 ligase involved in HSP90 inhibitor-induced SKP2 degradation, we performed proteomic analysis of SKP2-interacting proteins after STA-9090 and MG132 treatment. The putative E3 candidates were listed in Supplementary Fig. S7a. Unfortunately, knockdown of any one of these E3 candidates (RNF219, DTX3L, UBR5, RING1, RNF2, and AMFR) did not rescue HSP90 inhibitor-induced SKP2 degradation (Supplementary Fig. S7b-g), suggesting that none of these candidates are involved in the downregulation of SKP2 upon HSP90 inhibition. Taken together, these findings indicate that additional 
a

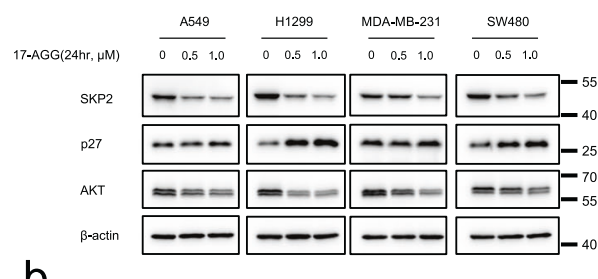

b

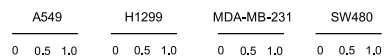

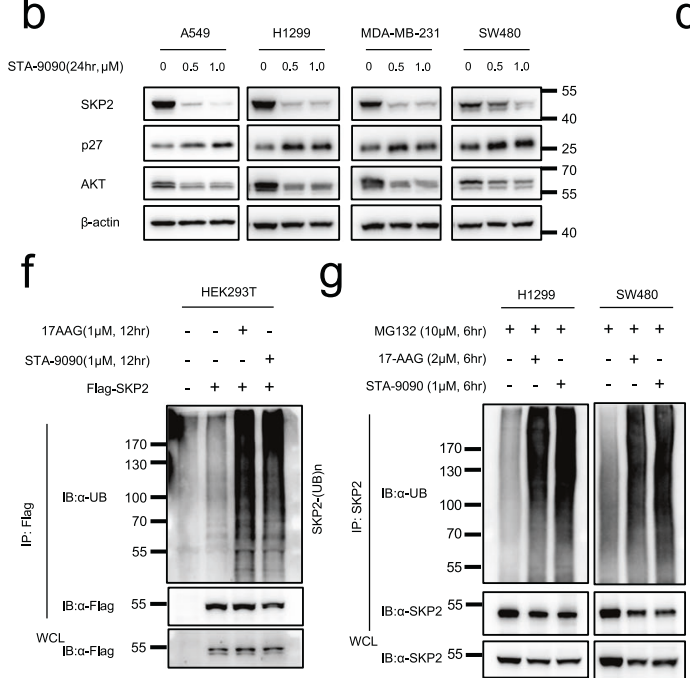

$\mathrm{h}$

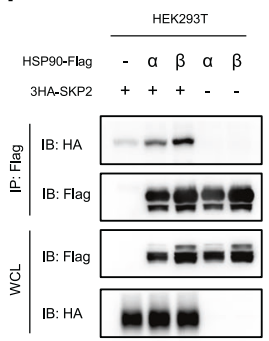

i GST-SKP2

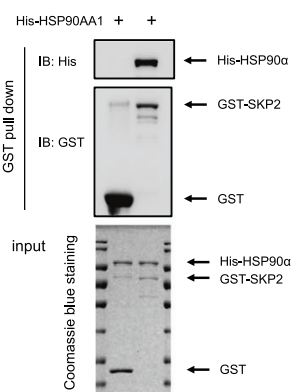

j

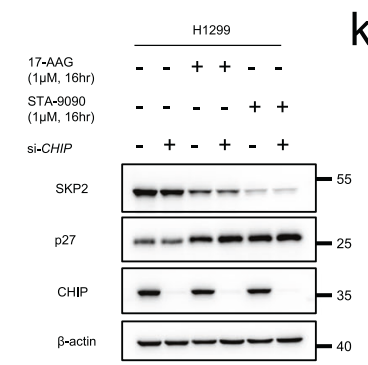

$\mathrm{k}$
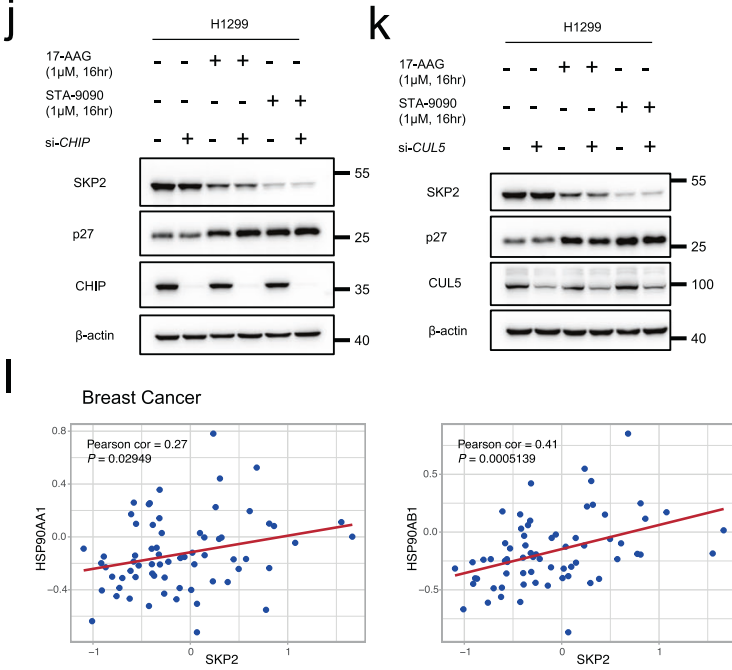

C

d
DMSO(hr) $\quad \begin{array}{lllllllllllll}0 & 2 & 4 & 6 & 8 & 10 & - & - & - & - & - & -\end{array}$

17-AAG(11M, hr)

CHX(500yg/mL, hr) $\quad 0 \begin{array}{llllllllllll}0 & 4 & 6 & 8 & 10 & 0 & 2 & 4 & 6 & 8 & 10\end{array}$
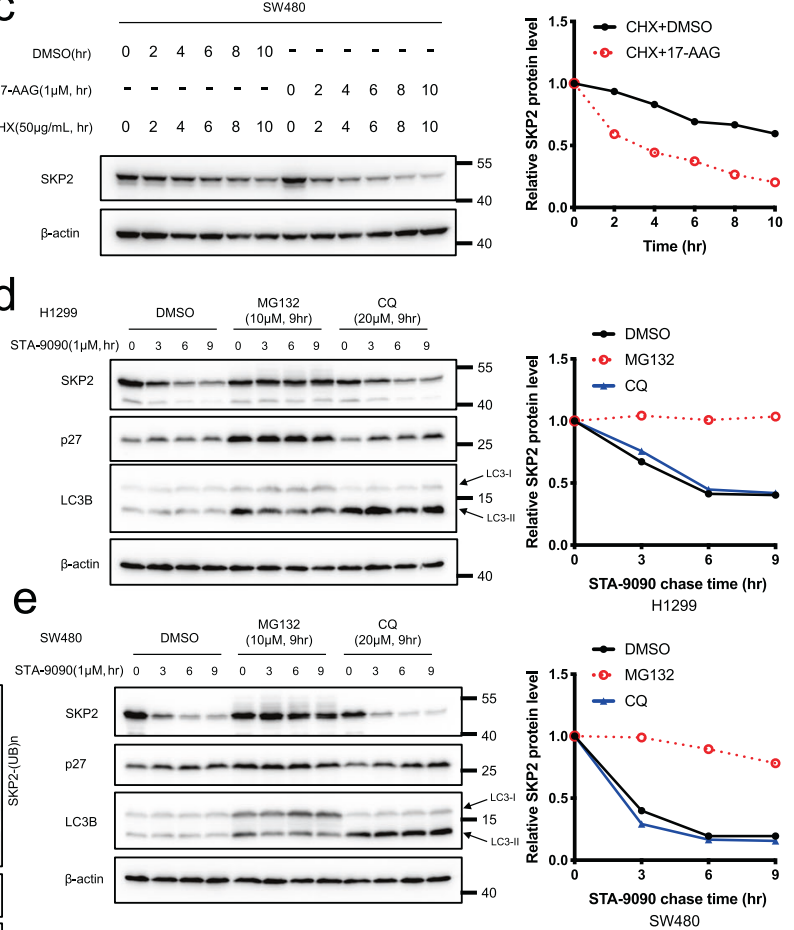

$\mathrm{m}$

GST-SKP2 $\quad-+$

His-HSP9OAB1 + +
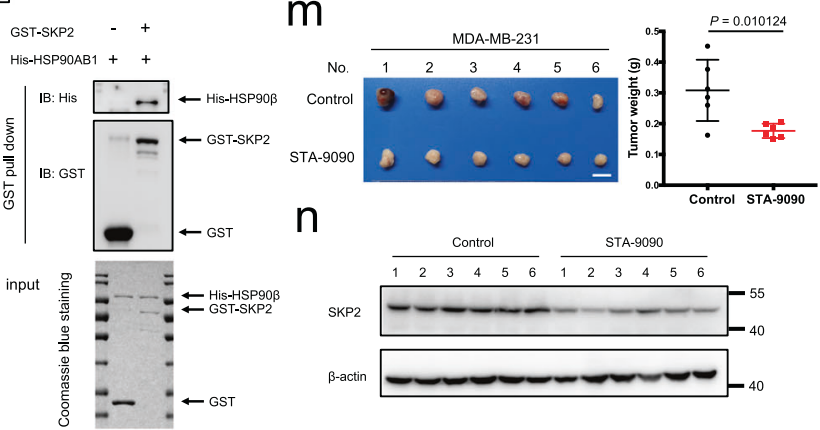

$\mathrm{n}$

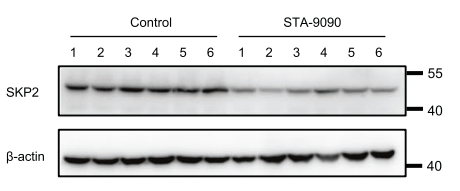

O

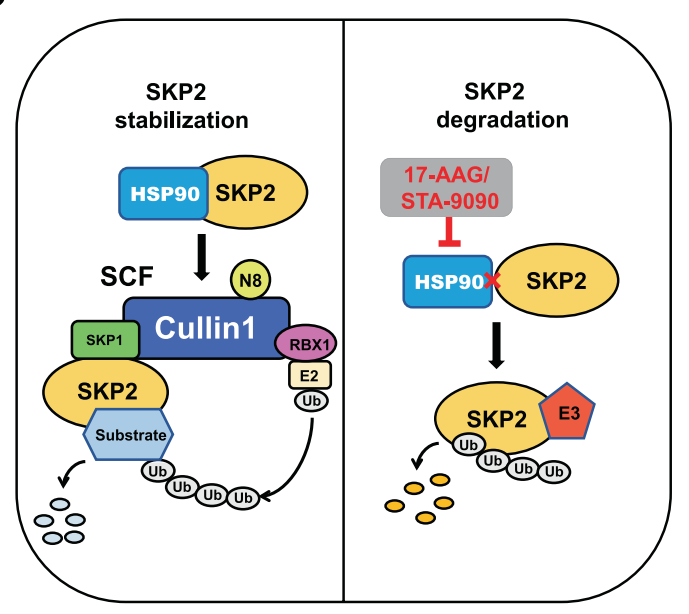

data of Breast Cancer ${ }^{5}$ (https://proteomic.datacommons.cancer. gov/pdc/) were examined, and Pearson's correlation analysis validated the positive correlation between HSP90 and SKP2 in breast cancer (Fig. 1I), indicating that the overexpression of HSP9O contributes to stabilizing SKP2 in tumors. 
Fig. 1 HSP90 inhibitors induce SKP2 ubiquitination and proteasomal degradation. a, b 17-AAG and STA-9090 treatment downregulated SKP2 in a dose-dependent manner in various cancer cells. c 17-AAG treatment promoted the degradation of SKP2 in SW480 cells. The band density of SKP2 was quantified by densitometric analysis, using ImageJ software (right panel). d, e STA-9090-induced SKP2 degradation was blocked by MG132 treatment, but not by chloroquine treatment. p27 was detected as a positive control of MG132 treatment, while LC3B was used as a positive control of CQ treatment. The band density of SKP2 was quantified by densitometric analysis, using ImageJ software (right panel). f 17AAG and STA-9090 induced the poly-ubiquitination of SKP2 in HEK293T cells transfected with Flag-tagged SKP2. g 17-AAG and STA-9090 induced the poly-ubiquitination of SKP2 in H1299 and SW480 cells. h SKP2 interacted with both HSP90 $\alpha$ and HSP90 3 . i SKP2 directly bound to HSP90 $\alpha$ and HSP90 $\beta$. j CHIP knockdown using siRNA did not compromise HSP90 inhibitor-induced SKP2 degradation. k CUL5 knockdown using siRNA did not compromise HSP90 inhibitor-induced SKP2 degradation. I Pearson's correlation analysis revealed the positive correlation between HSP90 and SKP2 in breast cancer. $\mathbf{m}$ Mice were sacrificed and tumor tissues were harvested and photographed. Scale bar $=1 \mathrm{~cm}$. The tumor weight was measured with an electronic scale on the sacrificed day. Student's $t$ test was used for the significance analysis. $\mathbf{n}$ Proteins were extracted from tumor tissues and subjected to immunoblotting analysis against SKP 2 with $\beta$-actin as a loading control. o A model of the possible mechanism underlying the regulation of SKP2 by HSP90 chaperone machinery.

Furthermore, we assessed the therapeutic potential of STA-9090 in MDA-MB-231 subcutaneous transplantation tumor model. As shown, STA-9090 treatment significantly inhibited tumor growth, as analyzed by tumor weight $(P<0.05$, Fig. $1 \mathrm{~m})$, and downregulated SKP2 expression in xenografted nude mice (Fig. 1n). Unexpectedly, p27 was not accumulated in mice tumor samples of STA-9090 treatment group (data not shown), which may due to the complicated role of HSP90 inhibitors and sophisticated regulation of p27 in vivo.

In conclusion, the present study demonstrates that SKP2 is regulated by HSP90 chaperone machinery and undergoes polyubiquitination targeted proteasomal degradation upon HSP90 inhibition (Fig. 10). Given that the overexpression of both HSP90 and SKP2 have been observed in a number of cancers, overexpressed HSP90 assists the folding and stabilization of SKP2 to promote cancer progression through mediating the degradation of tumor-suppressive substrates (Fig. 10).

\section{ACKNOWLEDGEMENTS}

This work was supported by the following funds: The Chinese Minister of Science and Technology grant (2016YFA0501800), National Natural Science Foundation of China (Grants 81625018, 81802743, and 81820108022), Program of Shanghai Academic/ Technology Research Leader (18XD1403800), Innovation Program of Shanghai Municipal Education Commission (2019-01-07-00-10-E00056), and National Thirteenth Five-Year Science and Technology Major Special Project for New Drug and Development (2017ZX09304001).

\section{ADDITIONAL INFORMATION}

Supplementary information The online version contains supplementary material available at https://doi.org/10.1038/s41392-021-00624-1.

Competing interests: The authors declare no competing interests. L.J. is the editorial board member of Signal Transduction and Targeted Therapy, but he has not been involved in the process of the manuscript handling.
Lili Cai ${ }^{1}$, Lihui $\mathrm{Li}^{1}$, Xihui Chen ${ }^{1}$ and Lijun Jia ${ }^{1}$

${ }^{1}$ Cancer Institute, Longhua Hospital, Shanghai University of Traditional Chinese Medicine, Shanghai, China These authors contributed equally: Lili Cai, Lihui Li Correspondence: Lijun Jia (ljjia@shutcm.edu.cn)

\section{REFERENCES}

1. Wang, Z., Liu, P., Inuzuka, H. \& Wei, W. Roles of F-box proteins in cancer. Nat. Rev. Cancer 14, 233-247 (2014).

2. Trepel, J., Mollapour, M., Giaccone, G. \& Neckers, L. Targeting the dynamic HSP90 complex in cancer. Nat. Rev. Cancer 10, 537-549 (2010).

3. Schopf, F. H., Biebl, M. M. \& Buchner, J. The HSP90 chaperone machinery. Nat. Rev. Mol. Cell Biol. 18, 345-360 (2017).

4. Gao, D. et al. Phosphorylation by Akt1 promotes cytoplasmic localization of Skp2 and impairs APCCdh1-mediated Skp2 destruction. Nat. Cell Biol. 11, 397-408 (2009).

5. Krug, K. et al. Proteogenomic landscape of breast cancer tumorigenesis and targeted therapy. Cell 183, 1436-1456.e1431 (2020).

\begin{abstract}
Open Access This article is licensed under a Creative Commons adaptation, distribution and reproduction in any medium or format, as long as you give appropriate credit to the original author(s) and the source, provide a link to the Creative Commons license, and indicate if changes were made. The images or other third party material in this article are included in the article's Creative Commons license, unless indicated otherwise in a credit line to the material. If material is not included in the article's Creative Commons license and your intended use is not permitted by statutory regulation or exceeds the permitted use, you will need to obtain permission directly from the copyright holder. To view a copy of this license, visit http://creativecommons. org/licenses/by/4.0/.
\end{abstract}

(c) The Author(s) 2021 